\title{
An information processing analysis of mental multiplication'
}

DONALD F. DANSEREAU AND LEE W. GREGG

CARNEGIE INSTITUTE OF TECHNOLOGY

A difficulty factor based on a count of the subprocesses normally involved in "paper and pencil" multiplication (e.g., "multiply," "add," "carry," and "hold") was found to be highly correlated with the solution times of problems solved mentally. Time for solution appeared to be independent of whether the subject did the problems silently or aloud.

The primary purpose of this study was to explore the characteristics of memory imbedded in a task of variable difficulty. Mental multiplication was chosen as the task because it is well structured, of flexible difficulty, and highly dependent on retention. Wiegand (1963) and Birren et al (1954) found that total time for mentally calculating a string of operations was a positively accelerated function of the count of the individual operations. Thomas (1963) developed a difficulty factor based on the summation of the logs of the sum of all digits in each single-digit operation. He found a linear relationship between the factor and total solution time. The hypothesis proposed in this paper takes the form of still another measure of difficulty. This measure, which will be henceforth called the "stepby-step" factor, is based on the sum of the singledigit operations involved in a "paper and pencil" solution of a multi-digit multiplication. In an information processing sense the single-digit operations ("multiply," "add," "holds," and "carry") are the primary processes. These processes are assumed to remain unchanged in all relevant task situations. In this case, the components are assumed to remain invariant, as to the amount of "difficulty" (time) contributed, over all problems. Also, a further assumption was made that each of the four components would contribute equally to the total difficulty factor.

\section{Method}

The S, a junior at the Carnegie Institute of Technology, was run under the following experimental conditions:

(1) Four sessions were conducted to determine a baseline; each of the sessions consisted of the following tasks:

(a) Five 2-digit by 3-digit multiplications were presented on a sheet of paper with appropriate space for solution. The $S$ was required to solve these problems in the conventional "paper and pencil" format. Verbalization of each step was required. (b) The final task was to solve mentally five to seven multiplication problems varying from $2 \times 2$ 's to $2 \times 4^{\prime} \mathrm{s}$. The problems were given to the $\mathrm{S}$ verbally (and repeated if necessary) by the experimenter. The $S$ was instructed to verbalize each step made during the solution process (the sessions were recorded on a Wollensak tape recorder). If the $S$ remained silent too long, the experimenter urged the $\mathrm{S}$ to talk. For each of these problems, a difficulty factor, such as described in the introduction, was developed.

(2) An incentive condition was introduced. If the $S$ worked a problem correctly in under half the time required for a problem of the same difficulty in Part 1 , he received $\$ 1.00$. If he worked it correctly in under $3 / 4$ of the time he received $\$ .25$. The $S$ was informed of the time limitations before each problem. Five sessions were run under these conditions. All sessions were conducted as in Part 1.

(3) The next six sessions were run under the conditions of Part 2. However, the $S$ was asked to do the problems silently rather than aloud and to report his answer only when in complete form.

(4) The final 10 sessions were run exactly according to Part 2.

Results

The data, in terms of total time per correct problem, were plotted against the proposed difficulty factor (Fig. 1 and 2). In each case a straight line fit was produced by the method of least squares; the regression lines account for approximately $90 \%$ of the variance. A test developed by Chow (1960) was run on the "Silent" and "Aloud" results to determine if these sets of data were representative of two distinct populations. The test did not approach significance. The per cent differences between the two regression lines were also found. These differences were $6.3 \%$ for the slopes and $7.0 \%$ for the $\mathrm{y}$ intercepts.

\section{Discussion}

The difficulty measure developed by Thomas (1963) was applied to the data. It also accurately predicted the solution times. Again, approximately $90 \%$ of the variance was accounted for. Both difficulty factors are, therefore, equally efficient, but the relative simplicity of the "step-by-step" measure seems to make it somewhat more desirable. It should be noted, however, that analysis of the S's protocols has revealed that the assumption that each type of operation contributes an equivalent amount of time is incorrect. It is clear that the "hold" and "carry" operations take more time per operation than the "add" and "multiply." Unfortunately the exact times involved could not be found due to overlap among operations.

The F-test, which was run on the differences between the aloud and silent data, did not disprove the possibility that both sets of data are subsets of the same population. 


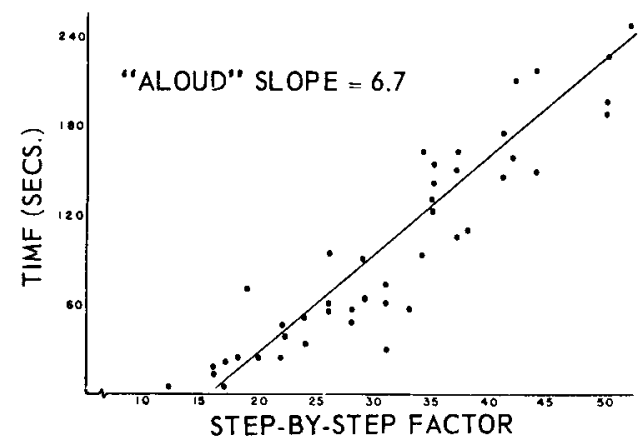

Fig. 1. Total solution time for the "Aloud" problems versus the Step-by-Step Difficulty Factor. The solid line is the least squares fitted line. $\mathbf{R}^{2}=.882$.

The percent errors calculated appear to be small enough to support this premise. The S's introspections reveal some interesting ideas on the behavior involved in these two situations. He stated that during the silent tasks the processing could be done much more rapidly than during the aloud stage. However, according to the $\mathrm{S}$, the fixation of the digits was more difficult under the silent condition. This led the $S$ to do the multiplications a number of times during the silent problems before moving on to the addition phase. A reasonable conclusion, at this point, might be that verbalization adds external memory aids at the expense of processing speed. The unusual finding, in this case, is that verbalization appears to add exactly as much as it detracts from the problem solving behavior. If the introspections of the $\mathrm{S}$ are ignored, as many people would suggest, another, perhaps more plausible, explanation of the homogeneity of the silent and aloud data can be offered. This explanation is based on a study by Landauer (1962); he finds that the rate of implicit speech is the same as that of explicit verbalization. His evidence stems from the comparison of groups reciting well-learned lists (e.g., the alphabet) implicit-

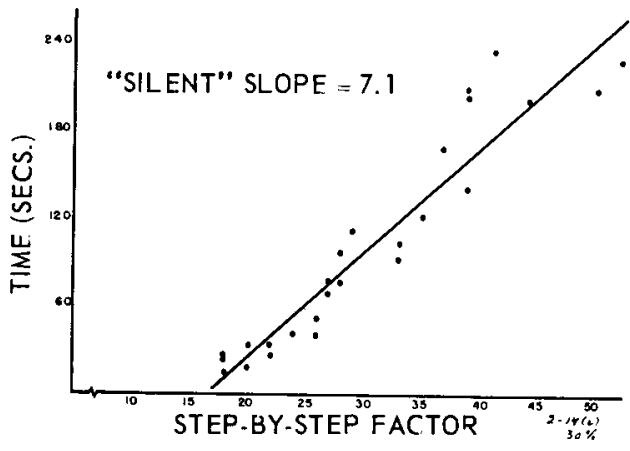

Fig. 2. Total solution time for the "Silent" problems versus the Step-by-Step Difficulty Factor. The solid line is the least squares fitted line. $\mathbf{R}^{2}=.914$.

ly and explicitly. Since simple calculations probably fall into this category, Landauer's results should be applicable to mental multiplication. It is clear, however, that the final resolution of the relative validity of these two hypotheses will have to await further empirical evidence.

\section{References}

Birren, J. E., Allen, W. R., \& Landau, H. G. The relation of problem length in simple addition to time required, probability of success, and age. J. Gerontol., 1954, 9, 150-161.

Chow, G. Tests of equality between sets of coefficients in two linear regressions. Econometrics, 1960, 28, 591-605.

Landauer, T. K. Rate of implicit speech. Percept. mot. Skills, $1962,15,646$.

Thomas, H. B. G. Communication theory and the constellation hypothesis of calculation. Quart. J. exp. Psychol., 1963, 15, 173-191.

Wiegand, K. L. Information theory and human behavior: uncertainty as a fundamental variable in information processing tasks. Technical Documentary Report No. AMRL-TDR-63-89, 1963, 91p.

\section{Note}

1. Supported by the Public Health Service, Department of Health, Education and Welfare, under Research Grant M-07722. This paper is based on the senior author's Master's thesis. 